\title{
The Phillip Island penguin parade (a mathematical treatment)*
}

\author{
Serena Dipierro ${ }^{\sharp 1,}$, Luca Lombardini ${ }^{2,3}$, Pietro Miraglio ${ }^{2,4}$, and Enrico Valdinoci ${ }^{1,2,5,6}$ \\ $\sharp$ Corresponding author \\ ${ }^{1}$ School of Mathematics and Statistics, University of Melbourne, Richard Berry Building, Parkville VIC 3010, Australia \\ ${ }^{2}$ Dipartimento di Matematica, Università degli studi di Milano, Via Saldini 50, 20133 Milan, Italy \\ ${ }^{3}$ Faculté des Sciences, Université de Picardie Jules Verne, 33, rue Saint-Leu, 80039 Amiens CEDEX 1, France \\ ${ }^{4}$ Departament de Matemàtica Aplicada I, Universitat Politècnica de Catalunya, Diagonal 647, 08028 Barcelona, Spain \\ ${ }^{5}$ Weierstraß Institut für Angewandte Analysis und Stochastik, Mohrenstraße 39, 10117 Berlin, Germany \\ ${ }^{6}$ Istituto di Matematica Applicata e Tecnologie Informatiche, Consiglio Nazionale delle Ricerche, Via Ferrata 1, 27100 Pavia, Italy
}

2010 Subject Classification: 92B05, 92B25, 37N25.

Keywords: Population dynamics, Eudyptula minor, Phillip Island, mathematical models.

\begin{abstract}
Penguins are flightless, so they are forced to walk while on land. In particular, they show rather specific behaviors in their homecoming, which are interesting to observe and to describe analytically.

In this paper, we present a simple mathematical formulation to describe the little penguins parade in Phillip Island.

We observed that penguins have the tendency to waddle back and forth on the shore to create a sufficiently large group and then walk home compactly together.

The mathematical framework that we introduce describes this phenomenon, by taking into account "natural parameters", such as the eye-sight of the penguins, their cruising speed and the possible "fear" of animals. On the one hand, this favors the formation of conglomerates of penguins that gather together, but, on the other hand, this may lead to the "panic" of isolated and exposed individuals.

The model that we propose is based on a set of ordinary differential equations. Due to the discontinuous behavior of the speed of the penguins, the mathematical treatment (to get existence and uniqueness of the solution) is based on a "stop-and-go" procedure.

We use this setting to provide rigorous examples in which at least some penguins manage to safely return home (there are also cases in which some penguins freeze due to panic).

To facilitate the intuition of the model, we also present some simple numerical simulations that can be compared with the actual movement of the penguins parade.
\end{abstract}

*Emails: sdipierro@unimelb.edu.au, luca.lombardini@unimi.it, pietro.miraglio@unimi.it, enrico@mat.uniroma3.it 


\section{Introduction}

The goal of this paper is to provide a simple, but rigorous, mathematical model which describes the formation of groups of penguins on the shore at sunset.

The results that we obtain are:

- The construction of a mathematical model to describe the formation of groups of penguins on the shore and their march towards their burrows; this model is based on systems of ordinary differential equations, with a number of degree of freedom which is variable in time (we show that the model admits a unique solution, which needs to be appropriately defined).

- Some rigorous mathematical results which provide sufficient conditions for a group of penguins to reach the burrows.

- Some numerical simulations which show that the mathematical model well predicts, at leasi1 at a qualitative level, the formation of clusters of penguins and their march towards the burrows; these simulations are easily implemented by images and videos.

The methodology used is based on:

1. direct observations on site, strict interactions with experts in biology and penguin ecology,

2. mathematical formulation of the problem and rigorous deductive arguments, and

3. numerical simulations.

In this introduction, we will describe the ingredients which lead to the construction of the model, presenting its basic features and also its limitations. Given the interdisciplinary flavor of the subject, it is not possible to completely split the biological discussion from the mathematical formulation, but we can mention that:

- The main mathematical equation is given in formula (1.1). Before (1.1), the main ingredients coming from live observations are presented. After (1.1), the mathematical quantities involved in the equation are discussed and elucidated.

- The existence and uniqueness theory for equation 1.1 is presented in Section 2

- Some rigorous mathematical results about equation (1.1) are given in Section 3 (roughly speaking, these are results which give sufficient conditions on the initial conditions of the system and on the external environment for the successful homecoming of the penguins, and their precise formulation requires the development of the mathematical framework in (1.1)).

- In Section 4 we present numerics, images and videos which favor the intuition and set the mathematical model of (1.1) into a concrete framework, which is easily comparable with the real-world phenomenon.

Before that, we think that it is important to describe our experience of the penguins parade in Phillip Island, both to allow the reader which is not familiar with the event to concretely take part in it, and to describe some peculiar environmental aspects which are crucial to understand our description (for instance, the weather in Phillip Island is completely different from the Antarctic one, so many of our considerations are meant to be limited to this particular habitat) - also, our personal experience in this bio-mathematical adventure is a crucial point, in our opinion, to describe how scientific curiosity can trigger academic activities. The reader who is not interested in the description of the penguins parade in Phillip Island can skip this part and go directly to Subsection 1.2

\footnotetext{
${ }^{1}$ It would be desirable to have empirical data about the formation of penguins clusters on the shore and their movements, in order to compare and adapt the model to experimental data and possibly give a quantitative description of concrete scenarios.
} 


\subsection{Description of the penguins parade}

An extraordinary event in the state of Victoria, Australia, consists in the march of the little penguins (whose scientific name is "Eudyptula minor") who live in Phillip Island. At sunset, when it gets too dark for the little penguins to hunt their food in the sea, they come out to return to their homes (which are small cavities in the terrain, that are located at some dozens of meters from the water edge).

As foreigners in Australia, our first touristic trip in the neighborhoods of Melbourne consisted in a one-day excursion to Phillip Island, enjoying the presence of wallabies, koalas and kangaroos, visiting some farms during the trip, walking on the spectacular empty beaches of the coast and - cherry on top - being delighted by the show of the little penguins parade.

Though at that moment we were astonished by the poetry of the natural exhibition of the penguins, later on, driving back to Melbourne in the middle of the night, we started thinking back to what we saw and attempted to understand the parade from a rational, and not only emotional, point of view (yet we believe that the rational approach was not diminishing but rather enhancing the sense of our intense experience). What follows is indeed the mathematical description that came out of the observations on site at Phillip Island, enriched by the scientific discussions we later had with penguin ecologists.

\subsection{Observed behaviors of penguins in the parade}

By watching the penguins parade in Phillip Island, it seemed to us that some simple features appeared in the very unusual pattern followed by the little penguins:

- Little penguins have the strong tendency to gather together in a sufficiently large number before starting their march home.

- They have the tendency to march on a straight line, compactly arranged in a cluster, or group.

- To make this group, they will move back and forth, waiting for other fellows or even going back to the sea if no other mate is around.

- If, by chance or by mistake, a little penguin remains isolated, (s)he can panic ${ }^{2}$ and this fact can lead to a complete ${ }^{3}$ freeze.

\footnotetext{
${ }^{2}$ In this paper, the use of terminology such as "panic" has to be intended in a strictly mathematical sense: namely, in the equation that we propose, there is a term which makes the velocity stop. The use of the word "panic" is due to the fact that this interruption in the penguin's movement is not due to physical impediments, but rather to the fact that no other penguin is in a sufficiently small neighborhood. Notice that it may be the case that a "penguin in panic" is not really in danger; simply, at a mathematical level, a quantified version of the notion of "isolation" leads the penguin to stop. For this reason, we think that the word "panic" is rather suggestive and actually sufficiently appropriate to describe a psychological attitude of the animal which could also turn out to be not necessarily convenient from the purely rational point of view: indeed, a "penguin in panic" risks to remain more exposed than a penguin that keeps moving (hence panic seems to be a good word to describe psychological uneasiness, with or without direct cause, that produces hysterical or irrational behavior).

It would be desirable to have further non-invasive tests to measure how the situation that we describe by the word "panic" is felt by the penguin at an emotional level (at the moment, we are not aware of experiments like this in the literature). Also, it would be highly desirable to have some precise experiments to determine how many penguins do not manage to return to their burrows within a certain time after dusk and stay either in the water or in the vicinity of the shore.

On the one hand, in our opinion, it is likely that rigorous experiments on site will demonstrate that the phenomenon for which an isolated penguin stops is rather uncommon in nature (and, when it happens, it may be unrelated to emotional feelings such as fear). On the other hand, our model is general enough to take into account the possibility that a penguin stops its march, and, at a quantitative level, we emphasized this "panic" feature in the pictures of Section 4 to make the situation visible.

The reader who does not want to take into account the panic function in the model can just set this function to be identically equal to 1 (the mathematical formulation of this remark will be given in footnote 7). In this particular case, our model will still exhibit the formation of groups of penguins moving together.

${ }^{3}$ Though no experimental test has been run on this phenomena, in the parade that we have seen live it indeed happened that one little penguin remained isolated from the others and "panic prevailed": even though (s)he was absolutely fit and no concrete obstacle was obstructing the motion, (s)he got completely stuck for half an hour and the staff of the Nature Park had to go and provide
} 
For a short video (courtesy of Phillip Island Nature Parks) of the little penguins parade, in which the formation of groups is rather evident, see e.g.

https://www.ma.utexas.edu/users/enrico/penguins/Penguins1.MOV

The simple features listed above are likely to be a consequence of the morphological structure of the little penguins and of the natural environment. As a matter of fact, little penguins are a marine-terrestrial species. They are highly efficient swimmers but possess a rather inefficient form of locomotion on land (indeed, flightless penguins, as the ones in Phillip Island, waddle, more than walk). At dusk, about 80 minutes after sunset according to the data in [13], little penguins return ashore after their fishing activity in the sea. Since their bipedal locomotion is slow and rather goofy (at least from the human subjective perception, but also in comparison with the velocity or agility that is well known to be typical of predators in nature), and the easily recognizable countershading of the penguins is likely to make them visible to predators, the transition between the marine and terrestrial environment may be particularly stressful $]^{4}$ for the penguins (see [9]) and this fact is probably related to the formation of penguins groups (see e.g. [2]). Thus, in our opinion, the rules that we have listed may be seen as the outcome of the difficulty of the little penguins to perform their transition from a more favorable environment to an habitat in which their morphology turns out to be suboptimal.

\subsection{Mathematical formulation}

To translate into a mathematical framework the simple observations on the penguins behavior that we listed in Subsection 1.2, we propose the following equation:

$$
\dot{p}_{i}(t)=\mathfrak{P}_{i}(p(t), w(t) ; t)\left(\varepsilon+\mathcal{V}_{i}(p(t), w(t) ; t)\right)+f\left(p_{i}(t), t\right) .
$$

Here, the following notation is used:

- The function $n:[0,+\infty) \rightarrow \mathbb{N}_{0}$, where $\mathbb{N}_{0}:=\mathbb{N} \backslash\{0\}$, is piecewise constant and nonincreasing, namely there exist a (possibly finite) sequence $0=t_{0}<t_{1}<\cdots<t_{j}<\ldots$ and integers $n_{1}>\cdots>$ $n_{j}>\ldots$ such that $n(t)=n_{j} \in \mathbb{N}_{0}$ for any $t \in\left(t_{j-1}, t_{j}\right)$.

- At time $t \geqslant 0$, there is a set of $n(t)$ groups of penguins $p(t)=\left(p_{1}(t), \ldots, p_{n(t)}(t)\right)$. That is, at time $t \in\left(t_{j-1}, t_{j}\right)$ there is a set of $n_{j}$ clusters of penguins $p(t)=\left(p_{1}(t), \ldots, p_{n_{j}}(t)\right)$.

- For any $i \in\{1, \ldots, n(t)\}$, the coordinate $p_{i}(t) \in \mathbb{R}$ represents the position of a group of penguins on the real line: each of these groups contains a certain number of little penguins, and this number is denoted by $w_{i}(t) \in \mathbb{N}_{0}$. We also consider the array $w(t)=\left(w_{1}(t), \ldots, w_{n(t)}(t)\right)$.

We assume that $w_{i}$ is piecewise constant, namely that $w_{i}(t)=\bar{w}_{i, j}$ for any $t \in\left(t_{j-1}, t_{j}\right)$, for some $\bar{w}_{i, j} \in \mathbb{N}_{0}$, namely the number of little penguins in each group remains constant, till the next

assistance. We stress again that the fact that the penguin stopped moving did not seem to be caused by any physical impediment (as confirmed to us by the Ranger on site), since no extreme environmental condition was occurring, the animal was not underweight, and was able to come out of the water and move effortlessly on the shore autonomously for about 15 meters, before suddenly stopping.

${ }^{4}$ At the moment, there seems to be no complete experimental evidence measuring the "danger perceived by the penguins", and, of course, the words "danger" and "fear" have to be intended - for the purposes of this paper, and strictly speaking - as a human interpretation, without experimental testing, and thus between "quotation marks". Nevertheless, given the swimming ability of the penguins and the environmental conditions, one may well conjecture that an area of high danger for a penguin is the one adjacent to the shore-line, since this is a habitat which provides little or no shelter, and it is also in a regime of reduced visibility.

As a matter of fact, we have been told that the Rangers in Phillip Island implemented a control on the presence of the foxes in the proximity of the shore, with the aim of limiting the number of possible predators.

Whether the penguins really feel an emotion comparable to what humans call "fear" or "panic" is not within the goals of this paper (it is of course also possible that, at a neurological level, the behavior of the penguins follows different patterns than human emotions). Nevertheless we use here the words "fear" and "danger" to give an easy-to-communicate justification of the mathematical model. Of course, any progress in the study of the emotional behavior of penguins would be highly desirable in this sense. 
penguins join the group at time $t_{j}$ (if, for the sake of simplicity, one wishes to think that initially all the little penguins are separated one from the other, one may also suppose that $w_{i}(t)=1$ for all $i \in\left\{1, \ldots, n_{1}\right\}$ and $t \in\left[0, t_{1}\right)$ ).

Up to renaming the variables, we suppose that the initial position of the groups is increasing with respect to the index, namely

$$
p_{1}(0)<\cdots<p_{n_{1}}(0) .
$$

- The parameter $\varepsilon \geqslant 0$ represents a drift velocity of the penguins towards their house, which is located at the point $H \in(0,+\infty)$.

- For any $i \in\{1, \ldots, n(t)\}$, the quantity $\mathcal{V}_{i}(p(t), w(t) ; t)$ represents the strategic velocity of the $i$ th group of penguins and it can be considered as a function with domain varying in time

$$
\mathcal{V}_{i}(\cdot, \cdot ; t): \mathbb{R}^{n(t)} \times \mathbb{N}^{n(t)} \rightarrow \mathbb{R}
$$

i.e.

$$
\mathcal{V}_{i}(\cdot, \cdot ; t): \mathbb{R}^{n_{j}} \times \mathbb{N}^{n_{j}} \rightarrow \mathbb{R} \quad \text { for any } t \in\left(t_{j-1}, t_{j}\right),
$$

and, for any $(\rho, w)=\left(\rho_{1}, \ldots, \rho_{n(t)}, w_{1}, \ldots, w_{n(t)}\right) \in \mathbb{R}^{n(t)} \times \mathbb{N}^{n(t)}$, it is of the form

$$
\mathcal{V}_{i}(\rho, w ; t):=\left(1-\mu\left(w_{i}\right)\right) m_{i}(\rho, w ; t)+v \mu\left(w_{i}\right) .
$$

In this setting, for any $(\rho, w)=\left(\rho_{1}, \ldots, \rho_{n(t)}, w_{1}, \ldots, w_{n(t)}\right) \in \mathbb{R}^{n(t)} \times \mathbb{N}^{n(t)}$, we have that

$$
m_{i}(\rho, w ; t):=\sum_{j \in\{1, \ldots, n(t)\}} \operatorname{sign}\left(\rho_{j}-\rho_{i}\right) w_{j} \mathfrak{s}\left(\left|\rho_{i}-\rho_{j}\right|\right),
$$

where $\mathfrak{s} \in \operatorname{Lip}([0,+\infty))$ is nonnegative and nonincreasing and, as usual, we denoted the "sign function" as

$$
\mathbb{R} \ni r \mapsto \operatorname{sign}(r):=\left\{\begin{array}{cl}
1 & \text { if } r>0, \\
0 & \text { if } r=0, \\
-1 & \text { if } r<0 .
\end{array}\right.
$$

Also, for any $\ell \in \mathbb{N}$, we set

$$
\mu(\ell):=\left\{\begin{array}{lc}
1 & \text { if } \ell \geqslant \kappa \\
0 & \text { if } \ell \leqslant \kappa-1,
\end{array}\right.
$$

for a fixed $\kappa \in \mathbb{N}$, with $\kappa \geqslant 2$, and $v>\varepsilon$.

In our framework, the meaning of the strategic velocity of the $i$ th group of penguins is the following:

- When the group of penguins is too small (i.e. it contains less than $\kappa$ little penguins), then the term involving $\mu$ vanishes, thus the strategic velocity reduces to the term given by $m_{i}$; this term, in turn, takes into account the position of the other groups of penguins. That is, each penguin is endowed with a "eye-sight" (i.e., the capacity of seeing the other penguins that are "sufficiently close" to them), which is modeled by the function $\mathfrak{s}$ (for instance, if $\mathfrak{s}$ is identically equal to 1 , then the penguin has a "perfect eye-sight"; if $\mathfrak{s}(r)=e^{-r^{2}}$, then the penguin sees close objects much better than distant ones; if $\mathfrak{s}$ is compactly supported, then the penguin does not see too far objects, etc.). Based on the position of the other mates that (s)he sees, the penguin has the tendency to move either forward or backward (the more penguins (s)he sees ahead, the more (s)he

\footnotetext{
${ }^{5}$ For concreteness, if $p_{i}(T)=H$ for some $T \geqslant 0$, we can set $p_{i}(t):=H$ for all $t \geqslant T$ and remove $p_{i}$ from the equation of motion - that is, the penguin has safely come back home and (s)he can go to sleep. In real life penguins have some social life before going to sleep, but we are not taking this under consideration for the moment.
} 
is inclined to move forward, the more penguins (s)he sees behind, the more (s)he is inclined to move backward, and nearby penguins weight more than distant ones, due to the monotonicity of $\mathfrak{s})$. This strategic tension coming from the position of the other penguins is encoded by the function $m_{i}$.

- When the group of penguins is sufficiently large (i.e. it contains at least $\kappa$ little penguins), then the term involving $\mu$ is equal to 1 ; in this case, the strategic velocity is $v$ (that is, when the group of penguins is sufficiently rich in population, its strategy is to move forward with cruising speed equal to $v$ ).

- The function $\mathfrak{P}_{i}(p(t), w(t) ; t)$ represents the panic that the $i$ th group of penguins fears in case of extreme isolation from the rest of the herd. Here, we take $\bar{d}>\underline{d}>0$, a nonincreasing ${ }^{6}$ function $\varphi \in$ $\operatorname{Lip}(\mathbb{R},[0,1])$, with $\varphi(r)=1$ if $r \leqslant \underline{d}$ and $\varphi(r)=0$ if $r \geqslant \bar{d}$, and, for any $\ell \in \mathbb{N}_{0}$,

$$
\mathfrak{w}(\ell):= \begin{cases}1 & \text { if } \ell \geqslant 2 \\ 0 & \text { if } \ell=1\end{cases}
$$

and we take as panic function $7^{7}$ the function with variable domain

$$
\mathfrak{P}_{i}(\cdot, \cdot ; t): \mathbb{R}^{n(t)} \times \mathbb{N}^{n(t)} \rightarrow[0,1],
$$

i.e.

$$
\mathfrak{P}_{i}(\cdot, \cdot ; t): \mathbb{R}^{n_{j}} \times \mathbb{N}^{n_{j}} \rightarrow[0,1] \quad \text { for any } t \in\left(t_{j-1}, t_{j}\right),
$$

given, for any $(\rho, w)=\left(\rho_{1}, \ldots, \rho_{n(t)}, w_{1}, \ldots, w_{n(t)}\right) \in \mathbb{R}^{n(t)} \times \mathbb{N}^{n(t)}$, by

$$
\mathfrak{P}_{i}(\rho, w ; t):=\max \left\{\mathfrak{w}\left(w_{i}\right), \max _{\substack{j \in\{1, \ldots, n(t)\} \\ j \neq i}} \varphi\left(\left|\rho_{i}-\rho_{j}\right|\right)\right\}
$$

The panic function describes the fact that, if the group gets scared, then it has the tendency to suddenly stop. This happens when the group contains only one element (i.e., $\mathfrak{w}_{i}=0$ ) and the other groups are far apart (at distance larger than $\bar{d}$ ).

Conversely, if the group contains at least two little penguins, or if there is at least another group sufficiently close (say at distance smaller than $\underline{d}$ ), then the group is self-confident, namely the function $\mathfrak{P}_{i}(p(t), w(t) ; t)$ is equal to 1 and the total intentional velocity of the group coincides with the strategic velocity.

Interestingly, the panic function $\mathfrak{P}_{i}$ may be independent of the eye-sight function $\mathfrak{s}$ : namely a little penguin can panic if (s)he feels alone and too much exposed, even if (s)he can see other little penguins (for instance, if $\mathfrak{s}$ is identically equal to 1 , the little penguin always sees the other members of the herd, still (s)he can panic if they are too far apart).

- The function $f \in \operatorname{Lip}(\mathbb{R} \times[0,+\infty))$ takes into account the environment. For a neutral environment, one has that this term vanishes. In practice, it may take into account the ebb and flow of the sea on the foreshore (where the little penguins parade starts), the possible ruggedness of the terrain, the presence of predators, etc. (as a variation, one can consider also a stochastic version of this term).

Given the interpretation above, equation (1.1) tries to comprise the pattern that we described in words and to set the scheme of motion of the little penguins into a mathematical framework.

\footnotetext{
${ }^{6}$ Here the notation "Lip" stands for bounded and Lipschitz continuous functions.

${ }^{7}$ The case of $\varphi$ identically equal to 1 can be also comprised in our setting. In this case, also $\mathfrak{P}_{i}$ is identically one (which corresponds to the case in which penguins do not panic).
} 


\subsection{Comparison with the existing literature}

We observe that, to the best of our knowledge, there is still no specific mathematical attempt to describe in a concise way the penguins parade. The mathematical literature of penguins has mostly focused on the description of the heat flow in the penguins feathers (see [4]), on the numerical analysis to mark animals for later identification (see [14]), on the statistics of the Magellanic penguins at sea (see [15]), on the hunting strategies of fishing penguins (see [7]), and on the isoperimetric arrangement of the Antarctic penguins to prevent the heat dispersion caused by the polar wind and on the crystal structures and solitary waves produced by such arrangements (see [6] and [11]). We remark that the climatic situation in Phillip Island is rather different from the Antarctic one and, given the very mild temperatures of the area, we do not think that heat considerations should affect too much the behavior and the moving strategies of the Victorian little penguins and their tendency to cluster seems more likely to be a defensive strategy against possible predators.

Though no mathematical formulation of the little penguins parade has been given till now, a series of experimental analysis has been recently performed on the specific environment of Phillip Island. We recall, in particular, [2], which describes the association of the little penguins in groups, by collecting data spanning over several years, [1], which describes the effect of fog on the orientation of the little penguins (which may actually do not come back home in conditions of poor visibility), [10] and [12], in which a data analysis is performed to show the fractal structure in space and time for the foraging of the little penguins, also in relation to Lévy flights and fractional Brownian motions.

For an exhaustive list of publications focused on the behavior of the little penguins of Phillip Island, we refer to the web page

https://www.penguins.org.au/conservation/research/publications/

This pages contains more than 160 publications related to the environment of Phillip Island, with special emphasis on the biology of little penguins.

\subsection{Discussion on the model proposed: simplifications, generalizations and further direc- tions of investigation}

We stress that the model proposed in (1.1) is of course a dramatic simplification of "reality". As often happens in science indeed, several simplifications have been adopted in order to allow a rigorous mathematical treatment and handy numerical computations: nevertheless the model is already rich enough to detect some specific features of the little penguins parade, such as the formation of groups, the oscillatory waddling of the penguins and the possibility that panic interferes with rationally more convenient motions. Moreover, our model is flexible enough to allow specific distinctions between the single penguins (for instance, with minor modification $\$ 8$, one can take into account the possibility that different penguins have a different eye-sight, that they have a different reaction to isolation and panic, or that they exhibit some specific social behavior that favors the formation of clusters selected by specific characteristics); similarly, the modeling of the habitat may also encode different possibilities (such as the burrows of the penguins being located in different places), and multi-dimensional models can be also constructed using similar ideas.

Furthermore, natural modifications lead to the possibility that one or a few penguins may leave an already formed group 9 (at the moment, for simplicity, we considered here the basic model in which, once a cluster is made up, it keeps moving without losing any of its elements - we plan to address in a future project in detail the case of groups which may also decrease the number of components, possibly in dependence of random fluctuations or social considerations among the members of the group).

\footnotetext{
${ }^{8}$ In particular, one can replace the quantities $v, \mathfrak{s}, \mu, \kappa, \varphi$ with $v_{i}, \mathfrak{s}_{i}, \mu_{i}, \kappa_{i}, \varphi_{i}$ if one wants to customize these features for every group.

${ }^{9}$ For instance, rather than forming one single group, the model can still consider the penguins of the cluster as separate elements, each one with its own peculiar behavior.
} 
In addition, for simplicity, in this paper we modeled each group to be located at a precise point: though this is not a completely unrealistic assumption (given that the scale of the penguin is much smaller than that of the beach), one can also easily modify this feature by locating a cluster in a region comparable to its size.

In future projects, we plan to introduce other more sophisticated models, also taking into account stochastic oscillations and optimization methods, and, on the long range, to use these models in a detailed experimental confrontation taking advantage of the automated monitoring systems under development in Phillip Island.

The model that we propose here is also flexible enough to allow quantitative modifications of all the parameters involved. This is quite important, since these parameters may vary due to different conditions of the

environment. For instance, the eye-sight of the penguins can be reduced by the fog (see [1]), and by the effect of moonlight and artificial light (see [13]).

Similarly, the number of penguins in each group and the velocity of the herd may vary due to structural changes of the beach: roughly speaking, from the empirical data, penguins typically gather into groups of 510 individuals (but we have also observed much larger groups forming on the beach) within 40 second intervals, see [2], but the way these groups are built varies year by year and, for instance, the number of individuals which always gather into the same group changes year by year in strong dependence with the breeding success of the season, see again [2]. Also, tidal phenomena may change the number of little penguins in each group and the velocity of the group, since the change of the beach width alters the perception of the risk of the penguins. For instance, a low tide produces a larger beach, with higher potential risk of predators, thus making the penguins gather in groups of larger size, see [9].

From the mathematical viewpoint, we remark that (1.1) does not follow into the classical framework of ordinary differential equations, since the right hand side of the equation is not Lipschitz continuous (and, in fact, it is not even continuous). This mathematical complication is indeed the counterpart of the real motion of the little penguins in the parade, which have the tendency to change their speed rather abruptly to maintain contact with the other elements of the herd. That is, on view, it does not seem unreasonable to model, as a simplification, the speed of the penguin as a discontinuous function, to take into account the sudden modifications of the waddling according to the position of the other penguins, with the conclusive aim of gathering together a sufficient number of penguins in a group which eventually will march concurrently in the direction of their burrows.

\subsection{Detailed organization of the paper}

The mathematical treatment of equation (1.1) that we provide in this paper is the following.

- In Section 2, we provide a notion of solution for which (1.1) is uniquely solvable in the appropriate setting. This notion of solution will be obtained by a "stop-and-go" procedure, which is compatible with the idea that when two (or more) groups of penguins meet, they form a new, bigger group which will move coherently in the sequel of the march.

- In Section 3, we discuss a couple of concrete examples in which the penguins are able to safely return home: namely, we show that there are "nice" conditions in which the strategy of the penguins allows a successful homecoming.

- In Section 4, we present a series of numerical simulations to compare our mathematical model with the real-world experience.

\section{Existence and uniqueness theory for equation (1.1)}

We stress that equation (1.1) does not lie within the setting of ordinary differential equations, since the right hand side is not Lipschitz continuous (due to the discontinuity of the functions $w$ and $m_{i}$, and in fact the right 
hand side also involves functions with domain varying in time). As far as we know, the weak formulations of ordinary differential equations as the ones of [3] do not take into consideration the setting of equation (1.1], so we briefly discuss here a direct approach to the existence and uniqueness theory for such equation. To this end, and to clarify our direct approach, we present two illustrative examples (see e.g. [5]).

Example 2.1. Setting $x:[0,+\infty) \rightarrow \mathbb{R}$, the ordinary differential equation

$$
\dot{x}(t)=\left\{\begin{array}{cc}
-1 & \text { if } x(t) \geqslant 0 \\
1 & \text { if } x(t)<0
\end{array}\right.
$$

is not well posed. Indeed, taking an initial datum $x(0)<0$, it will evolve with the formula $x(t)=t+x(0)$ for any $t \in[0,-x(0)]$ till it hits the zero value. At that point, equation 2.1 would prescribe a negative velocity, which becomes contradictory with the positive velocity prescribed to the negative coordinates.

Example 2.2. The ordinary differential equation

$$
\dot{x}(t)=\left\{\begin{array}{cc}
-1 & \text { if } x(t)>0, \\
0 & \text { if } x(t)=0, \\
1 & \text { if } x(t)<0
\end{array}\right.
$$

is similar to the one in (2.1), in the sense that it does not fit into the standard theory of ordinary differential equations, due to the lack of continuity of the right hand side. But, differently from the one in (2.1), it can be set into an existence and uniqueness theory by a simple "reset" algorithm.

Namely, taking an initial datum $x(0)<0$, the solution evolves with the formula $x(t)=t+x(0)$ for any $t \in[0,-x(0)]$ till it hits the zero value. At that point, equation 2.2 would prescribe a zero velocity, thus a natural way to continue the solution is to take $x(t)=0$ for any $t \in[-x(0),+\infty)$ (similarly, in the case of positive initial datum $x(0)>0$, a natural way to continue the solution is $x(t)=-t+x(0)$ for any $t \in[0, x(0)]$ and $x(t)=0$ for any $t \in[x(0),+\infty)$ ). The basic idea for this continuation method is to flow the equation according to the standard Cauchy theory of ordinary differential equations for as long as possible, and then, when the classical theory breaks, "reset" the equation with respect of the datum at the break time (this method is not universal and indeed it does not work for 2.1), but it produces a natural global solution for (2.2).

In the light of Example 2.2, we now present a framework in which equation 1.1 possesses a unique solution (in a suitable "reset" setting). To this aim, we first notice that the initial number of groups of penguins is fixed to be equal to $n_{1}$ and each group is given by a fixed number of little penguins packed together (that is, the number of little penguins in the $i$ th initial group being equal to $\bar{w}_{i, 1}$ and $i$ ranges from 1 to $n_{1}$ ). So, we set $\bar{w}_{1}:=\left(\bar{w}_{1,1}, \ldots, \bar{w}_{n_{1}, 1}\right)$ and $\overline{\mathfrak{w}}_{i, 1}=\mathfrak{w}\left(\bar{w}_{i, 1}\right)$, where $\mathfrak{w}$ was defined in 1.6 . For any $\rho=\left(\rho_{1}, \ldots, \rho_{n_{1}}\right) \in$ $\mathbb{R}^{n_{1}}$, let also

$$
\mathfrak{P}_{i, 1}(\rho):=\max \left\{\overline{\mathfrak{w}}_{i, 1}, \max _{\substack{j \in\left\{1, \ldots, n_{1}\right\} \\ j \neq i}} \varphi\left(\left|\rho_{i}-\rho_{j}\right|\right)\right\} .
$$

The reader may compare this definition with the one in 1.7$]$. For any $i \in\left\{1, \ldots, n_{1}\right\}$ we also set

$$
\bar{\mu}_{i, 1}:=\mu\left(\bar{w}_{i, 1}\right),
$$

where $\mu$ is the function defined in 1.5 , and, for any $\rho=\left(\rho_{1}, \ldots, \rho_{n_{1}}\right) \in \mathbb{R}^{n_{1}}$,

$$
\bar{m}_{i, 1}(\rho):=\sum_{j \in\left\{1, \ldots, n_{1}\right\}} \operatorname{sign}\left(\rho_{j}-\rho_{i}\right) \bar{w}_{j, 1} \mathfrak{s}\left(\left|\rho_{i}-\rho_{j}\right|\right) .
$$

This definition has to be compared with (1.4). Recalling (1.2) we also set

$$
\mathcal{D}_{1}:=\left\{\rho=\left(\rho_{1}, \ldots, \rho_{n_{1}}\right) \in \mathbb{R}^{n_{1}} \text { s.t. } \rho_{1}<\cdots<\rho_{n_{1}}\right\} .
$$


We remark that if $\rho \in \mathcal{D}_{1}$ then

$$
\bar{m}_{i, 1}(\rho)=\sum_{j \in\left\{i+1, \ldots, n_{1}\right\}} \bar{w}_{j, 1} \mathfrak{s}\left(\left|\rho_{i}-\rho_{j}\right|\right)-\sum_{j \in\{1, \ldots, i-1\}} \bar{w}_{j, 1} \mathfrak{s}\left(\left|\rho_{i}-\rho_{j}\right|\right)
$$

and therefore

$$
\bar{m}_{i, 1}(\rho) \text { is bounded and Lipschitz for any } \rho \in \mathcal{D}_{1} \text {. }
$$

Then, we set

$$
\mathcal{V}_{i, 1}(\rho):=\left(1-\bar{\mu}_{i, 1}\right) \bar{m}_{i, 1}(\rho)+v \bar{\mu}_{i, 1} .
$$

This definition has to be compared with the one in (1.3). Notice that, in view of (2.4), we have that

$$
\mathcal{V}_{i, 1}(\rho) \text { is bounded and Lipschitz for any } \rho \in \mathcal{D}_{1} .
$$

So, we set

$$
G_{i, 1}(\rho, t):=\mathfrak{P}_{i, 1}(\rho)\left(\varepsilon+\mathcal{V}_{i, 1}(\rho)\right)+f\left(\rho_{i}, t\right) .
$$

From (2.3) and (2.5), we have that $G_{i, 1}$ is bounded and Lipschitz in $\mathcal{D}_{1} \times[0,+\infty)$. Consequently, from the global existence and uniqueness of solutions of ordinary differential equations, we have that there exist $t_{1} \in$ $(0,+\infty]$ and a solution $p^{(1)}(t)=\left(p_{1}^{(1)}(t), \ldots, p_{n_{1}}^{(1)}(t)\right) \in \mathcal{D}_{1}$ of the Cauchy problem

$$
\left\{\begin{array}{l}
\dot{p}_{i}^{(1)}(t)=G_{i, 1}\left(p^{(1)}(t), t\right) \\
p^{(1)}(0) \quad \text { given in } \mathcal{D}_{1}
\end{array} \quad \text { for } t \in\left(0, t_{1}\right),\right.
$$

and

$$
p^{(1)}\left(t_{1}\right) \in \partial \mathcal{D}_{1},
$$

see e.g. Theorem 1.4.1 in [8].

The solution of (1.1) will be taken to be $p^{(1)}$ in $\left[0, t_{1}\right)$, that is, we set $p(t):=p^{(1)}(t)$ for any $t \in\left[0, t_{1}\right)$. We also set that $n(t):=n_{1}$ and $w(t):=\left(\bar{w}_{1,1}, \ldots, \bar{w}_{n_{1}, 1}\right)$. With this setting, we have that $p$ is a solution of equation (1.1) in the time range $t \in\left(0, t_{1}\right)$ with prescribed initial datum $p(0)$. Condition (2.6) allows us to perform our "stop-and-go" reset procedure as follows: we denote by $n_{2}$ the number of distinct points in the set $\left\{p_{1}^{(1)}\left(t_{1}\right), \ldots, p_{n_{1}}^{(1)}\left(t_{1}\right)\right\}$. Notice that (2.6) says that if $t_{1}$ is finite then $n_{2} \leqslant n_{1}-1$ (namely, at least two penguins have reached the same position). In this way, the set of points $\left\{p_{1}^{(1)}\left(t_{1}\right), \ldots, p_{n_{1}}^{(1)}\left(t_{1}\right)\right\}$ can be identified by the set of $n_{2}$ distinct points, that we denote by $\left\{p_{1}^{(2)}\left(t_{1}\right), \ldots, p_{n_{2}}^{(2)}\left(t_{1}\right)\right\}$, with the convention that

$$
p_{1}^{(2)}\left(t_{1}\right)<\cdots<p_{n_{2}}^{(2)}\left(t_{1}\right) .
$$

For any $i \in\left\{1, \ldots, n_{2}\right\}$, we also set

$$
\bar{w}_{i, 2}:=\sum_{\substack{j \in\left\{1, \ldots, n_{1}\right\} \\ p_{j}^{(1)}\left(t_{1}\right)=p_{i}^{(2)}\left(t_{1}\right)}} \bar{w}_{j, 1}
$$

This says that the new group of penguins indexed by $i$ contains all the penguins that have reached that position at time $t_{1}$.

Thus, having the "new number of groups", that is $n_{2}$, the "new number of little penguins in each group", that is $\bar{w}_{2}=\left(\bar{w}_{1,2}, \ldots, \bar{w}_{n_{2}, 2}\right)$, and the "new initial datum", that is $p^{(2)}\left(t_{1}\right)=\left(p_{1}^{(2)}\left(t_{1}\right), \ldots, p_{n_{2}}^{(2)}\left(t_{1}\right)\right)$, we can solve a new differential equation with these new parameters, exactly in the same way as before, and keep iterating this process. 
Indeed, recursively, we suppose that we have found $t_{1}<t_{2}<\cdots<t_{k}, p^{(1)}:\left[0, t_{1}\right] \rightarrow \mathbb{R}^{n_{1}}, \ldots$, $p^{(k)}:\left[0, t_{k}\right] \rightarrow \mathbb{R}^{n_{k}}$ and $\bar{w}_{1} \in \mathbb{N}_{0}^{n_{1}}, \ldots, \bar{w}_{k} \in \mathbb{N}_{0}^{n_{k}}$ such that, setting

$$
p(t):=p^{(j)}(t) \in \mathcal{D}_{j}, \quad n(t):=n_{j} \quad \text { and } \quad w(t):=\bar{w}_{j} \quad \text { for } t \in\left[t_{j-1}, t_{j}\right) \text { and } j \in\{1, \ldots, k\},
$$

one has that $p$ solves (1.1) in each interval $\left(t_{j-1}, t_{j}\right)$ for $j \in\{1, \ldots, k\}$, with the "stop condition"

$$
p^{(j)}\left(t_{j}\right) \in \partial \mathcal{D}_{j},
$$

where

$$
\mathcal{D}_{j}:=\left\{\rho=\left(\rho_{1}, \ldots, \rho_{n_{j}}\right) \in \mathbb{R}^{n_{j}} \text { s.t. } \rho_{1}<\cdots<\rho_{n_{j}}\right\} .
$$

Then, since $p^{(k)}\left(t_{k}\right) \in \partial \mathcal{D}_{k}$, if $t_{k}$ is finite, we find $n_{k+1} \leqslant n_{k}-1$ such that the set of points $\left\{p_{1}^{(k)}\left(t_{k}\right), \ldots, p_{n_{k}}^{(k)}\left(t_{k}\right)\right\}$ coincides with a set of $n_{k+1}$ distinct points, that we denote by $\left\{p_{1}^{(k+1)}\left(t_{k}\right), \ldots, p_{n_{k}}^{(k+1)}\left(t_{k}\right)\right\}$, with the convention that

For any $i \in\left\{1, \ldots, n_{k+1}\right\}$, we se ${ }^{10}$

$$
p_{1}^{(k+1)}\left(t_{k}\right)<\cdots<p_{n_{k}}^{(k+1)}\left(t_{k}\right) .
$$

$$
\bar{w}_{i, k+1}:=\sum_{\substack{j \in\left\{1, \ldots, n_{k}\right\} \\ p_{j}^{(k)}\left(t_{k}\right)=p_{i}^{(k+1)}\left(t_{k}\right)}} \bar{w}_{j, k} .
$$

Let also $\overline{\mathfrak{w}}_{i, k+1}=\mathfrak{w}\left(\bar{w}_{i, k+1}\right)$. Then, for any $i \in\left\{1, \ldots, n_{k+1}\right\}$ and any $\rho=\left(\rho_{1}, \ldots, \rho_{n_{k+1}}\right) \in \mathbb{R}^{n_{k+1}}$, we set

$$
\mathfrak{P}_{i, k+1}(\rho):=\max \left\{\overline{\mathfrak{w}}_{i, k+1}, \max _{\substack{j \in\left\{1, \ldots, n_{k+1}\right\} \\ j \neq i}} \varphi\left(\left|\rho_{i}-\rho_{j}\right|\right)\right\} \text {. }
$$

For any $i \in\left\{1, \ldots, n_{k+1}\right\}$ we also define

$$
\bar{\mu}_{i, k+1}:=\mu\left(\bar{w}_{i, k+1}\right),
$$

where $\mu$ is the function defined in 1.5 and, for any $\rho \in \mathbb{R}^{n_{k+1}}$,

$$
\bar{m}_{i, k+1}(\rho):=\sum_{j \in\left\{1, \ldots, n_{k+1}\right\}} \operatorname{sign}\left(\rho_{j}-\rho_{i}\right) \bar{w}_{j, k+1} \mathfrak{s}\left(\left|\rho_{i}-\rho_{j}\right|\right) .
$$

We notice that $\bar{m}_{i, k+1}(\rho)$ is bounded and Lipschitz for any $\rho \in \mathcal{D}_{k+1}:=\left\{\rho=\left(\rho_{1}, \ldots, \rho_{n_{k+1}}\right) \in\right.$ $\mathbb{R}^{n_{k+1}}$ s.t. $\left.\rho_{1}<\cdots<\rho_{n_{k+1}}\right\}$.

We also define

$$
\mathcal{V}_{i, k+1}(\rho):=\left(1-\bar{\mu}_{i, k+1}\right) \bar{m}_{i, k+1}(\rho)+v \bar{\mu}_{i, k+1}
$$

and

$$
G_{i, k+1}(\rho, t):=\mathfrak{P}_{i, k+1}(\rho)\left(\varepsilon+\mathcal{V}_{i, k+1}(\rho)\right)+f\left(\rho_{i}, t\right) .
$$

In this way, we have that $G_{i, k+1}$ is bounded and Lipschitz in $\mathcal{D}_{k+1} \times[0,+\infty)$ and so we find the next solution $p^{(k+1)}(t)=\left(p_{1}^{(k+1)}(t), \ldots, p_{n_{k+1}}^{(k+1)}(t)\right) \in \mathcal{D}_{k+1}$ in the interval $\left(t_{k}, t_{k+1}\right)$, with $p^{(k+1)}\left(t_{k+1}\right) \in$ $\partial \mathcal{D}_{k+1}$, by solving the ordinary differential equation

$$
\dot{p}_{i}^{(k+1)}(t)=G_{i, k+1}\left(p^{(k+1)}(t), t\right) .
$$

This completes the iteration argument and provides the desired notion of solution for equation (1.1).

\footnotetext{
${ }^{10}$ It is useful to observe that, in light of (2.7),
}

$$
\sum_{i \in\left\{1, \ldots, n_{k+1}\right\}} \bar{w}_{i, k+1}=\sum_{i \in\left\{1, \ldots, n_{k}\right\}} \bar{w}_{i, k},
$$

which says that the total number of little penguins remains always the same (more precisely, the sum of all the little penguins in all groups is constant in time). 


\section{Examples of safe return home}

Here, we provide some sufficient conditions for the penguins to reach their home, located at the point $H$ (let us mention that, in the parade that we saw live, one little penguin remained stuck into panic and did not manage to return home - so, giving a mathematical treatment of the case in which the strategy of the penguins turns out to be successful somehow reassured us on the fate of the species).

To give a mathematical framework of the notion of homecoming, we introduce the function

$$
[0,+\infty) \ni t \mapsto \mathcal{N}(t):=\sum_{\substack{j \in\{1, \ldots, n(t)\} \\ p_{j}(t)=H}} w_{j}(t) .
$$

In the setting of footnote 5 , the function $\mathcal{N}(t)$ represents the number of penguins that have safely returned home at time $t$.

For counting reasons, we also point out that the total number of penguins is constant and given by

$$
\mathcal{M}:=\sum_{j \in\{1, \ldots, n(0)\}} w_{j}(0)=\sum_{j \in\{1, \ldots, n(t)\}} w_{j}(t),
$$

for any $t \geqslant 0$ (recall footnote 10 ).

The first result that we present says that if at some time the group of penguins that stay further behind gathers into a group of at least two elements, then all the penguins will manage to eventually return home. The mathematical setting goes as follows:

Theorem 3.1. Let $t_{o} \geqslant 0$ and assume that

$$
\varepsilon+\inf _{(r, t) \in \mathbb{R} \times\left[t_{o},+\infty\right)} f(r, t) \geqslant \iota
$$

for some $\iota>0$, and

$$
w_{1}\left(t_{o}\right) \geqslant 2 .
$$

Then, there exists $T \in\left[t_{o}, t_{o}+\frac{H-p_{1}\left(t_{o}\right)}{\iota}\right]$ such that

$$
\mathcal{N}(T)=\mathcal{M}
$$

Proof. We observe that $w_{1}(t)$ is nondecreasing in $t$, thanks to (2.7), and therefore (3.2) implies that $w_{1}(t) \geqslant$ 2 for any $t \geqslant t_{o}$. Consequently, from (1.6), we obtain that $\mathfrak{w}\left(w_{1}(t)\right)=1$ for any $t \geqslant t_{o}$. This and (1.7) give that $\mathfrak{P}_{1}(\rho, w(t) ; t)=1$ for any $t \geqslant t_{o}$ and any $\rho \in \mathbb{R}^{n(t)}$. Accordingly, the equation of motions in (1.1) gives that, for any $t \geqslant t_{o}$,

$$
\dot{p}_{1}(t)=\varepsilon+\mathcal{V}_{1}(p(t), w(t) ; t)+f\left(p_{1}(t), t\right) \geqslant \varepsilon+f\left(p_{1}(t), t\right) \geqslant \iota,
$$

thanks to 3.1. That is, for any $j \in\{1, \ldots, n(t)\}$,

$$
p_{j}(t) \geqslant p_{1}(t) \geqslant \min \left\{H, p_{1}\left(t_{o}\right)+\iota\left(t-t_{o}\right)\right\},
$$

which gives the desired result.

A simple variation of Theorem 3.1 says that if, at some time, a group of little penguins reaches a sufficiently large size, then all the penguins in this group (as well as the ones ahead) safely reach their home. The precise statement (whose proof is similar to the one of Theorem 3.1, up to technical modifications, and is therefore omitted) goes as follows: 
Theorem 3.2. Let $t_{o} \geqslant 0$ and assume that

$$
\varepsilon+v+\inf _{(r, t) \in \mathbb{R} \times\left[t_{o},+\infty\right)} f(r, t) \geqslant \iota
$$

for some $\iota>0$, and

$$
w_{j_{o}}\left(t_{o}\right) \geqslant \kappa,
$$

for some $j_{o} \in\left\{1, \ldots, n\left(t_{o}\right)\right\}$.

Then, there exists $T \in\left[t_{o}, t_{o}+\frac{H-p_{j_{o}}\left(t_{o}\right)}{\iota}\right]$ such that

$$
\mathcal{N}(T) \geqslant \sum_{j \in\left\{j_{o}, \ldots, n\left(t_{o}\right)\right\}} w_{j}\left(t_{o}\right)
$$

\section{Pictures, videos and numerics}

In this section, we present some simple numerical experiments to facilitate the intuition at the base of the model presented in (1.1). These simulations may actually be easily compared with the "real life" experience and indeed they show some of the typical treats of the little penguins parade, such as the oscillations and sudden change of direction, the gathering of the penguins into clusters and the possibility that some elements of the herd remain isolated and panic, either ${ }^{11}$ on the land or in the sea.

In our simulations, for the sake of simplicity, we considered 20 penguins returning to their burrows from the shore - some of the penguins may start their trip from the sea (that occupies the region below level 0 in the simulations) in which waves and currents may affect the movements of the animals. The pictures that we produce have the time variable on the horizontal axis and the space variable on the vertical axis (with the burrow of the penguins community set at level 4 for definiteness). The pictures are, somehow, selfexplanatory. For instance, in Figure 1, we present a case in which, fortunately, all the little penguins manage to safely return home, after having gathered into groups: as a matter of fact, in the first of these pictures all the penguins safely reach home together at the same time (after having rescued the first penguin, who stayed still for a long period due to isolation and panic); on the other hand, the second of these pictures shows that a first group of penguins, which was originated by the animals that were on the land at the initial time, reaches home slightly before the second group of penguins, which was originated by the animals that were in the sea at the initial time (notice also that the motion of the penguins in the sea appears to be affected by waves and currents).

We also observe a different scenario depicted in Figure 2 (with two different functions to represent the currents in the sea): in this situation, a big group of 18 penguins gathers together (collecting also penguins who were initially in the water) and safely returns home. Two penguins remain isolated in the water, and they keep slowly moving towards their final destination (that they eventually reach after a longer time).

Similarly, in Figure 3, almost all the penguins gather into a single group and reach home, while two penguins get together in the sea, they come to the shore and slowly waddle towards their final destination, and one single penguin remains isolated and panics in the water, moved by the currents.

The situation in Figure 4 is slightly different, since the last penguin at the beginning moves towards the others, but (s)he does not manage to join the forming group by the time the other penguins decide to move consistently towards their burrows - so, unfortunately this last penguin, in spite of the initial effort, finally remains stuck in the water.

In Figure 5, all the penguins reach their burrows, with the exception of the last two ones: at the time we end the simulation, one penguin is stuck on the shore, due to panic, and another one is very slowly approaching

\footnotetext{
${ }^{11}$ The possibility that a penguin remains isolated also in the sea may actually occur in the real-world experience, as demonstrated by the last penguin in the herd on the video (courtesy of Phillip Island Nature Parks) available online at the webpage https://www.ma.utexas.edu/users/enrico/penguins/Penguins2.MOV
} 

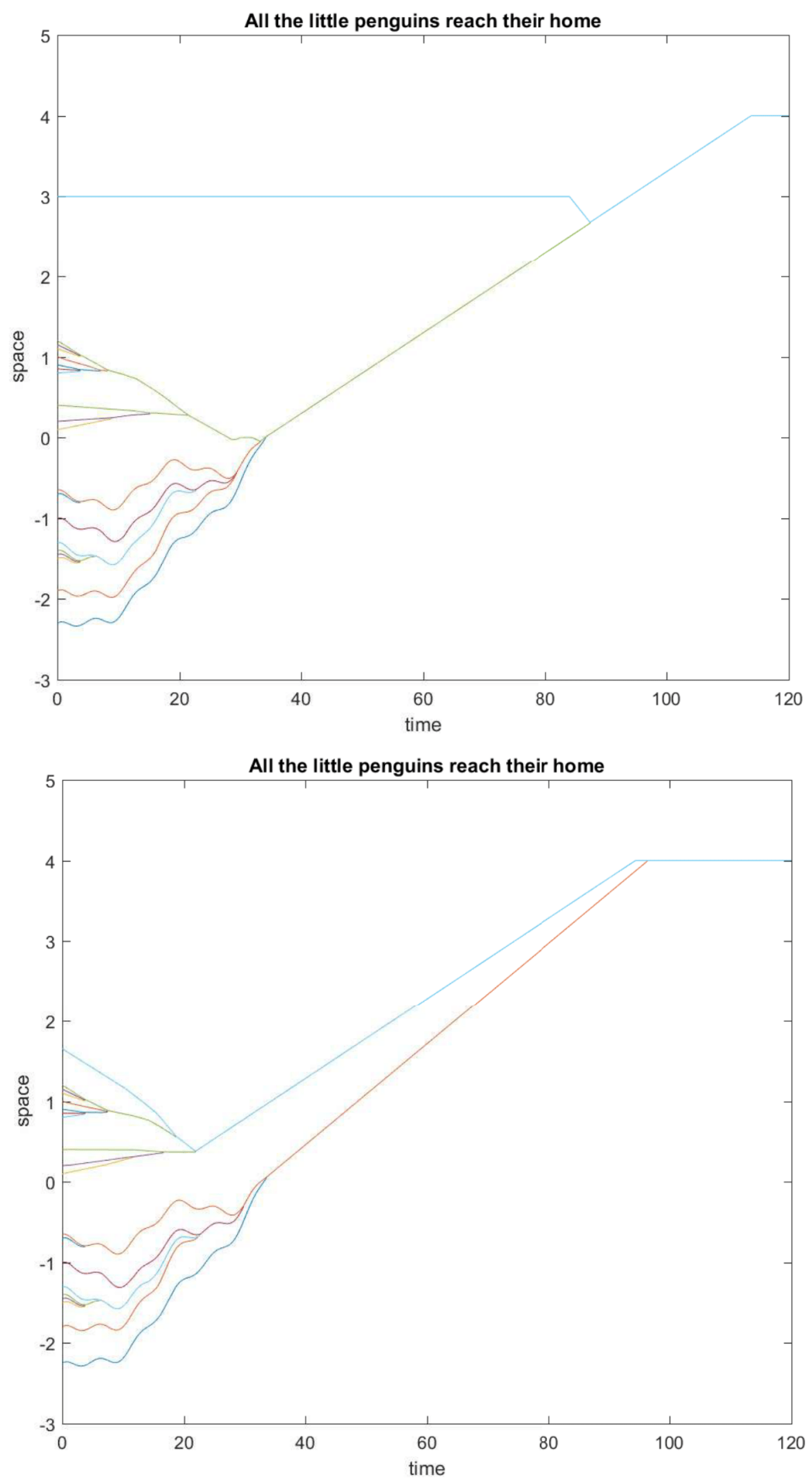

Figure 1: All the little penguins safely return home. 

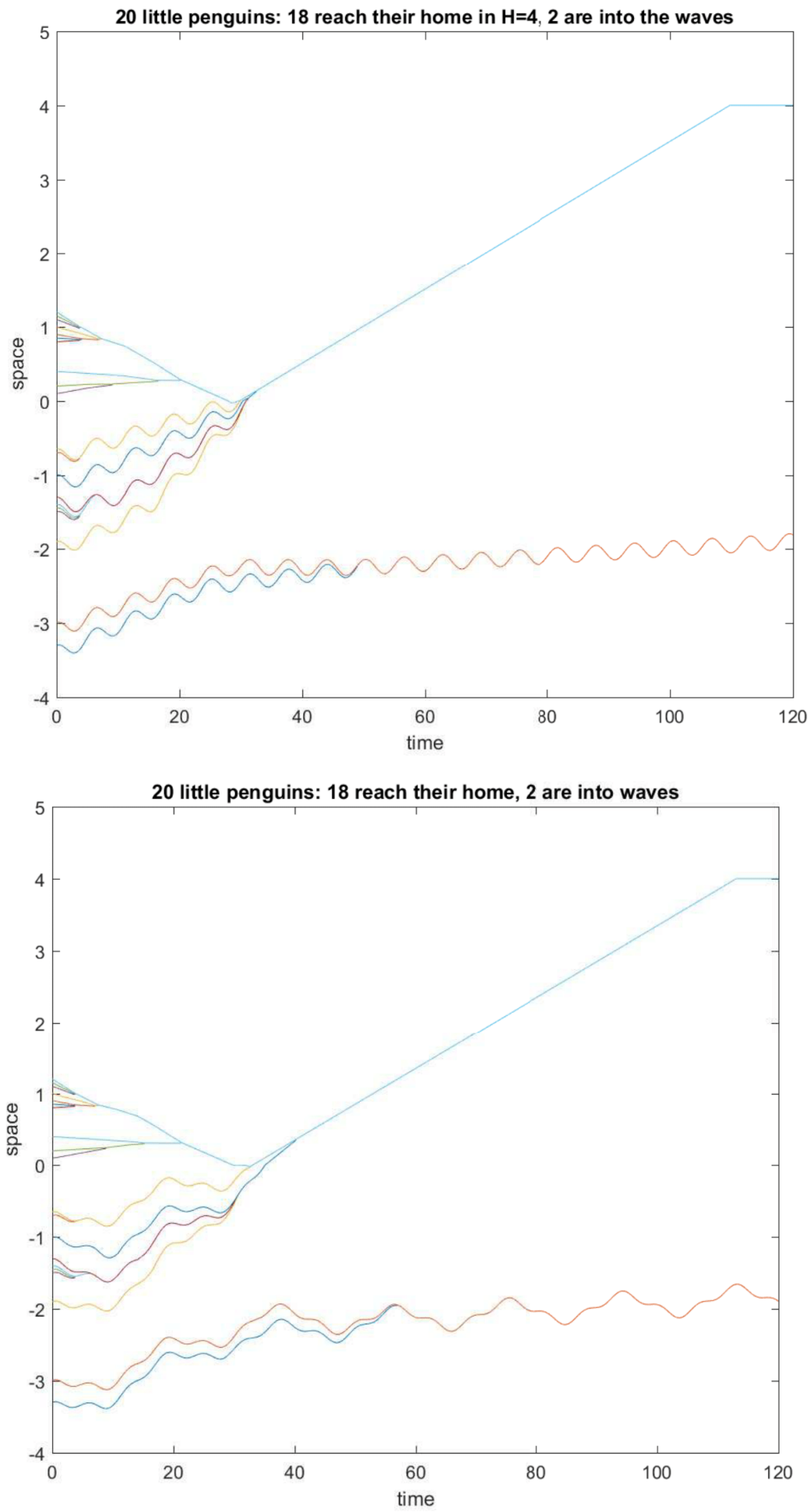

Figure 2: Two penguins are still in the water after a long time. 


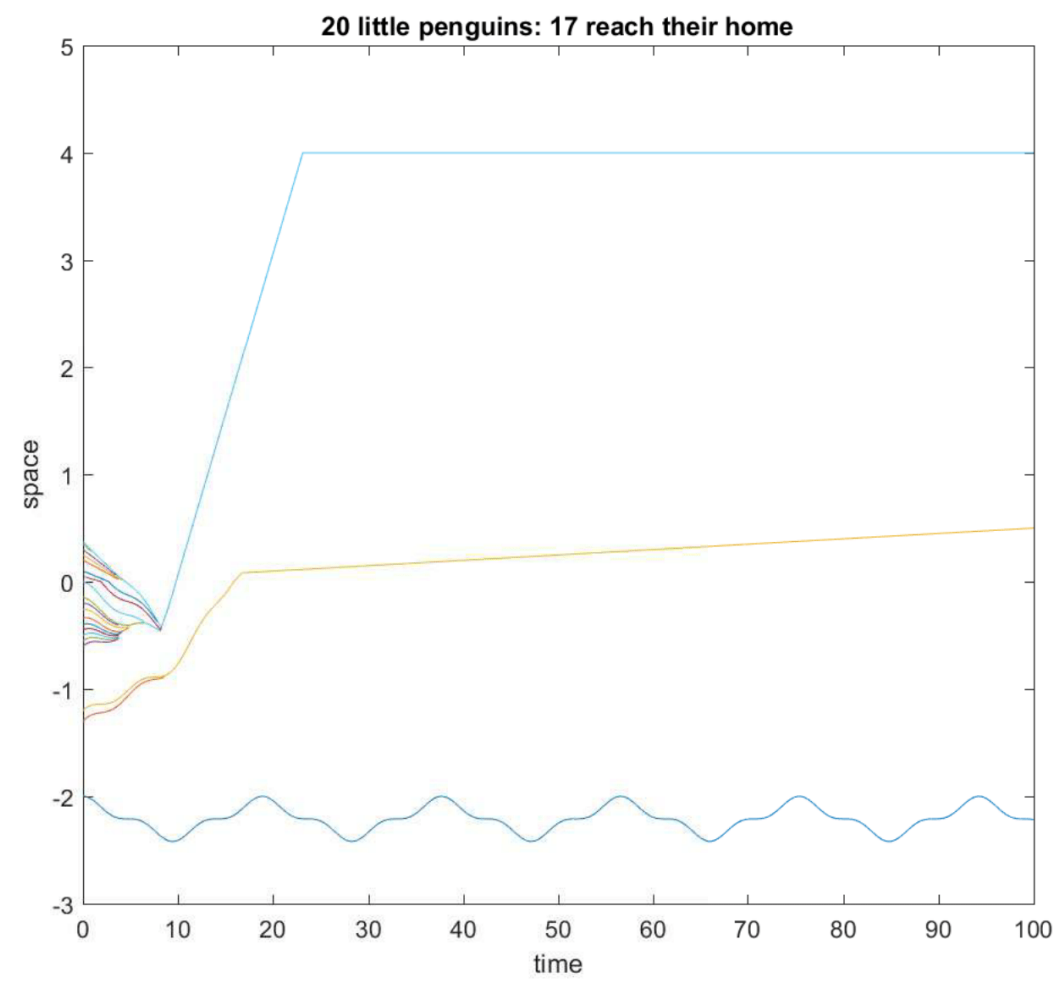

Figure 3: One penguin is stuck in the water.

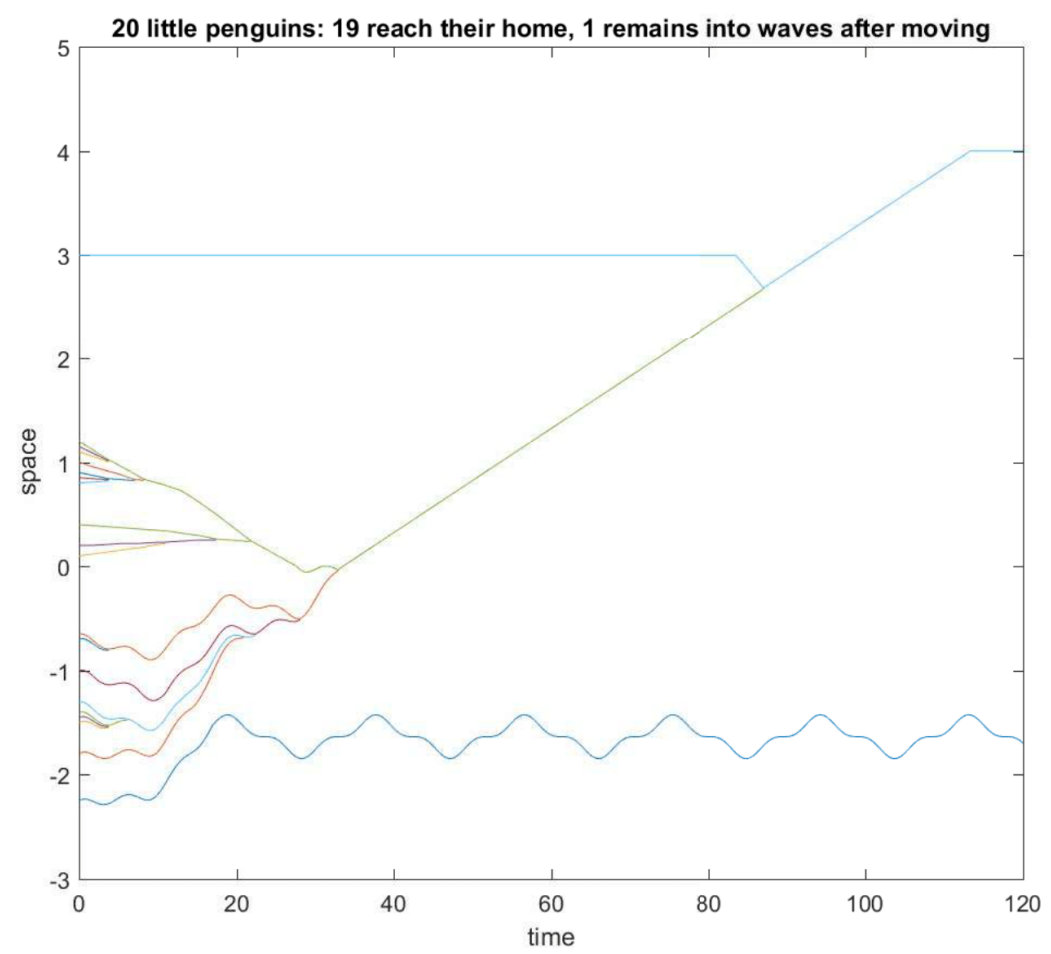

Figure 4: One penguin moves towards the others but remains stuck in the water. 


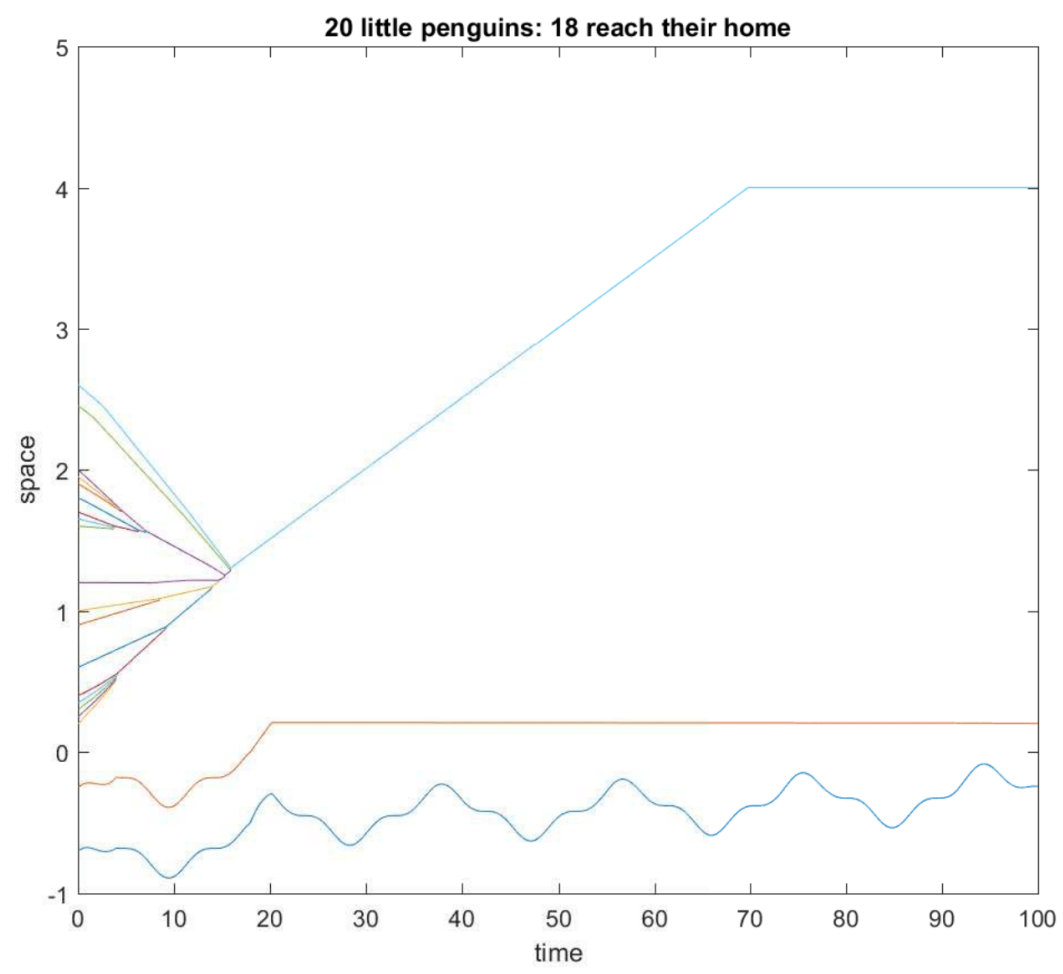

Figure 5: One penguin freezes on the shore, another stays in the water.

the beach, but (s)he is still in the water (small modifications of the initial conditions and of the wave function may lead to different future outcomes, namely either the last penguin is able to reach the shore and happily meet the other mate to waddle together home, or the strong current may prevent the last penguin to reach the beach, in which case also the penguin in front would remain stuck).

With simple modifications of the function $f$, one can also consider the case in which the waves of the sea change with time and their influence may become more (or less) relevant for the swimming of the little penguins: as an example of this feature, see Figure 6

Finally, we recall that, in the setting of Section 1, once a group of little penguins is created, then it moves consistently altogether. This is of course a simplifying assumption, and it might happen in reality that one or a few penguins leave a large group after its formation - perhaps because one penguin is slower than the other penguins of the group, perhaps because (s)he gets distracted by other events on the beach, or simply because (s)he feels too exposed being at the side of the group and may prefer to form a new group in which (s)he finds a more central and protected position. Though we plan to describe this case in detail in a forthcoming project (also possibly in light of morphological and social considerations and taking into account a possible randomness in the system), we stress that natural modifications can be implemented inside the setting of Section 1 to take into account also this feature. For simple and concrete examples, see Figure 7, in which several cases are considered (e.g., one of the little penguins leaving the group gets stuck, or goes back into the water, or meets another little penguin, and so on).

The situation in which one little penguin seems to think about leaving an already formed group can be observed in the video (courtesy of Phillip Island Nature Parks)

https://www.ma.utexas.edu/users/enrico/penguins/Penguins2.MOV

(see in particular the behavior of the second penguin from the bottom, i.e. the last penguin of the already formed large cluster).

We point out that all these pictures have been easily obtained by short programs in MathLab. As an example, 

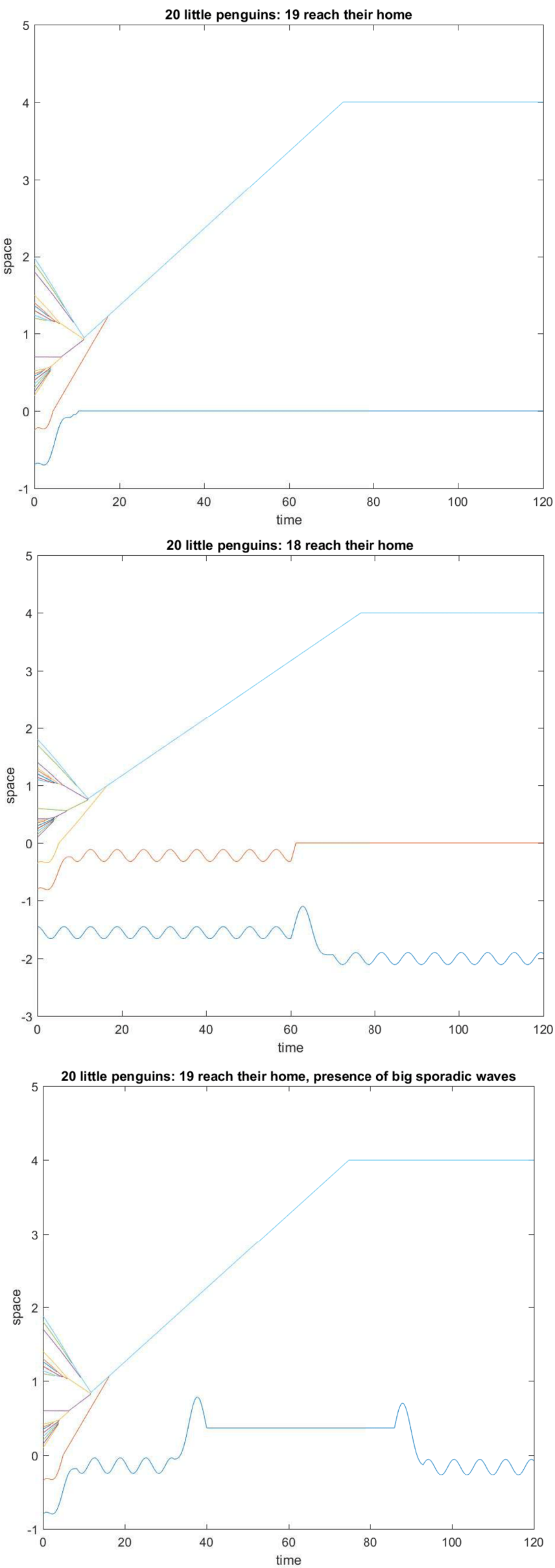

Figure 6: Effect of the waves on the movement of the penguins in the sea. 

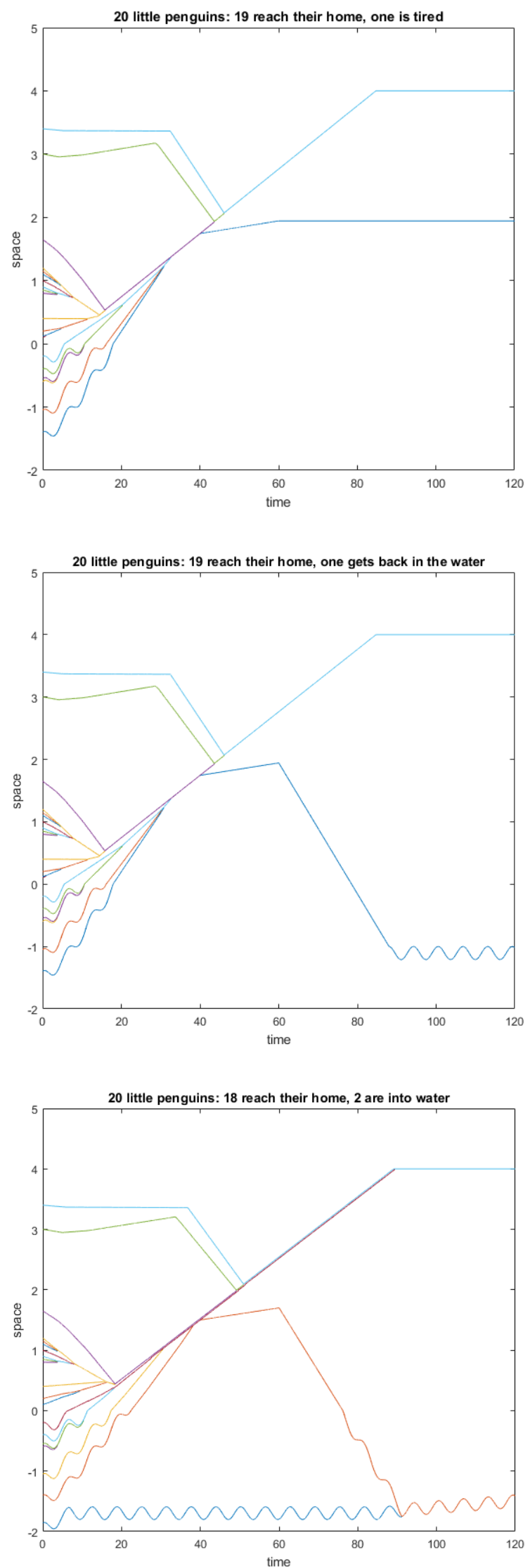

Figure 7: A modification: one little penguin may leave the group. 
we posted one of the source codes of these programs on the webpage

https://www.ma.utexas.edu/users/enrico/penguins/cononda.txt

and all the others are available upon request (the simplicity of these programs shows that the model in (1.1) is indeed very simple to implement numerically, still producing sufficiently "realistic" results in terms of cluster formation and cruising speed of the groups).

Also, these pictures can be easily translated into animations. Simple videos that we have obtained by these numerics are available from the webpage

https://www.ma.utexas.edu/users/enrico/penguins/VID/

\section{Acknowledgements}

This work has been supported by the ERC grant $\varepsilon$ (Elliptic Pde's and Symmetry of Interfaces and Layers for Odd Nonlinearities) and the PRIN grant 201274FYK7 (Critical Point Theory and Perturbative Methods for Nonlinear Differential Equations). Part of this paper has been written on the occasion of a very pleasant visit of the second and third authors to the University of Melbourne. We thank Claudia Bucur and Carina Geldhauser for their interesting comments on a preliminary version of this manuscript. We would also like to thank Andre Chiaradia for a very instructive conversation and for sharing the bibliographic information and some videos of the little penguins parade. Videos credit to Phillip Island Nature Parks.

\section{References}

[1] A. Chiaradia, J. McBride, T. Murray, and P. Dann, Effect of fog on the arrival time of little penguins Eudyptula minor: a clue for visual orientation?, Journal of Ornithology 148 (2007), no. 2, 229-233, DOI 10.1007/s10336-007-0125-5.

[2] T. Daniel, A. Chiaradia, M. Logan, G. Quinn, and R. Reina, Synchronized group association in little penguins, Eudyptula Minor, Animal behaviour 74 (2007), no. 5, 1241-1248, DOI 10.1016/j.anbehav.2007.01.029.

[3] R. J. DiPerna and P.-L. Lions, Ordinary differential equations, transport theory and Sobolev spaces, Invent. Math. 98 (1989), no. 3, 511-547, DOI 10.1007/BF01393835. MR1022305

[4] N. Du, J. Fan, H. Wu, S. Chen, and Y. Liu, An improved model of heat transfer through penguin feathers and down, J. Theoret. Biol. 248 (2007), no. 4, 727-735, DOI 10.1016/j.jtbi.2007.06.020. MR2899094

[5] A. F. Filippov, Differential equations with discontinuous righthand sides, Mathematics and its Applications (Soviet Series), vol. 18, Kluwer Academic Publishers Group, Dordrecht, 1988. Translated from the Russian. MR1028776

[6] R. C. Gerum, B. Fabry, C. Metzner, M. Beaulieu, A. Ancel, and D. P. Zitterbart, The origin of traveling waves in an emperor penguin huddle, New Journal of Physics 15 (2013), no. 12, 125022.

[7] Y. Gheraibia and A. Moussaoui, Penguins Search Optimization Algorithm (PeSOA), in Recent Trends in Applied Artificial Intelligence: 26th International Conference on Industrial, Engineering and Other Applications of Applied Intelligent Systems, IEA/AIE 2013, Amsterdam, The Netherlands, June 17-21, 2013, Springer Berlin Heidelberg, 2013.

[8] Q. Kong, A short course in ordinary differential equations, Universitext, Springer, Cham, 2014. MR3244289

[9] S. M. Laaksonen, A. Chiaradia, and R. D. Reina, Behavioural plasticity of a multihabitat animal, the little penguin, Eudyptula minor, in response to tidal oscillations on its interhabitat transitions, Preprint (2016). 
[10] A. J. J. Macintosh, L. Pelletier, A. Chiaradia, A. Kato, and Y. Ropert-Coudert, Temporal fractals in seabird foraging behaviour: diving through the scales of time, Sci Rep. 3 (2013), no. 1884, 1-10, DOI 10.1038/srep01884.

[11] R. Ragonnet, R. Jumentier, and B. Beckermann, La marche de l'empereur, Matapli 102 (2013), 71-82 (French). MR3235841

[12] A. M. Reynolds, Y. Ropert-Coudert, A. Kato, A. Chiaradia, and A. J. J. MacIntosh, A priority-based queuing process explanation for scale-free foraging behaviours, Animal Behaviour 108 (2015), 67-71, DOI 10.1016/j.anbehav.2015.07.022.

[13] A. Rodríguez, A. Chiaradia, P. Wasiak, L. Renwick, and P. Dann, Waddling on the Dark Side: Ambient Light Affects Attendance Behavior of Little Penguins, J. Biol. Rhythms 31 (2016), no. 2, 194-204, DOI $10.1177 / 0748730415626010$.

[14] L. A. Sidhu, E. A. Catchpole, and P. Dann, Modelling banding effect and tag loss for Little Penguins Eudyptula minor, ANZIAM J. Electron. Suppl. 52 (2010), no. (C), C206-C221. MR2926716

[15] E. A. Skewgar, Behavior of Magellanic penguins at sea, ProQuest LLC, Ann Arbor, MI, 2009. Thesis (Ph.D.)-University of Washington. MR2718048 